\title{
Association between tumour necrosis factor- $\alpha$ inhibitors and risk of serious infections in people with inflammatory bowel disease: nationwide Danish cohort study
}

\author{
Nynne Nyboe Andersen, ${ }^{1}$ Björn Pasternak, ${ }^{1}$ Nina Friis-Møller, ${ }^{2}$ Mikael Andersson, ${ }^{1}$ Tine Jess ${ }^{1}$
}

'Department of Epidemiology

Research, Statens Serum

Institute, Artillerivej 5, 2300

Copenhagen, Denmark

${ }^{2}$ Department of Infectious

Disease, Odense University

Hospital, Odense, Denmark

Correspondence to: N Nyboe

Andersennyna@ssi.dk

Additional material is published

online only. To view please visit

the journal online (http://dx.doi.

org/10.1136/bmj.h2809)

Cite this as: BMJ 2015;350:h2809

doi: 10.1136/bmj.h2809

Accepted: 19 May 2015

\section{ABSTRACT}

OBJECTIVE

To investigate whether people with inflammatory bowel disease treated with tumour necrosis factor- $\alpha$ (TNF- $\alpha$ ) inhibitors are at increased risk of serious infections.

DESIGN

Nationwide register based propensity score matched cohort study.

SETTING

Denmark, 2002-12.

\section{PARTICIPANTS}

The background cohort eligible for matching comprised 52392 people with inflammatory bowel disease, aged 15 to 75 years, of whom 4300 were treated with TNF- $\alpha$ inhibitors. To limit confounding, a two stage matching method was applied; firstly matching on age, sex, disease duration, and inflammatory bowel disease subtype, and secondly matching on propensity scores (1:1 ratio); this yielded 1543 people treated with TNF- $\alpha$ inhibitors and 1543 untreated to be included in the analyses.

\section{MAIN OUTCOME MEASURES}

The main outcome was any serious infection, defined as a diagnosis of infection associated with hospital admission. Cox regression was used to estimate hazard ratios for two risk periods ( 90 and 365 days after the start of TNF- $\alpha$ inhibitor treatment). Hazard ratios of site specific serious infections were obtained solely for the 365 days risk period.

RESULTS

Within the 90 days risk period, 51 cases of infection were observed in users of TNF- $\alpha$ inhibitors (incidence rate $14 / 100$ person years), compared with 33 cases in non-users ( $9 / 100$ person years), yielding a hazard ratio

\section{WHAT IS ALREADY KNOWN ON THIS TOPIC}

Tumour necrosis factor- $\alpha$ (TNF- $\alpha$ ) inhibitors are highly effective in the treatment of several immune mediated diseases, including inflammatory bowel disease

However, the risk of potential adverse events, including serious infections, remains a concern

Meta-analyses and population based studies investigating the association between TNF- $\alpha$ inhibitors and serious infections in people with inflammatory bowel disease are few and have yielded ambiguous results

\section{WHAT THIS STUDY ADDS}

This nationwide cohort study suggests that treatment of inflammatory bowel disease with TNF- $\alpha$ inhibitors is associated with an increased risk of serious infections within the first three months of the start of treatment and a subsequent decline in risk

of 1.63 (95\% confidence interval 1.01 to 2.63 ). Within the risk period of 365 days, the hazard ratio was 1.27 (0.92 to 1.75). In analyses of site specific infections, the hazard ratio was above 2 for several of the subgroups but only reached statistical significance for skin and soft tissue infections (2.51, 1.23 to 5.12).

\section{CONCLUSIONS}

This nationwide propensity score matched cohort study suggests an increased risk of serious infections associated with use of TNF- $\alpha$ inhibitors within the first 90 days of starting treatment and a subsequent decline in risk. This calls for increased clinical awareness of potential infectious complications among people with inflammatory bowel disease using these drugs, especially early in the course of treatment.

\section{Introduction}

Tumour necrosis factor- $\alpha$ (TNF- $\alpha$ ) inhibitors are highly effective in the treatment of several immune mediated diseases, including inflammatory bowel diseases. The most commonly used TNF- $\alpha$ inhibitors in people with inflammatory bowel disease are infliximab, adalimumab, and certolizumab pegol. All three drugs are approved for the treatment of Crohn's disease, whereas only infliximab and adalimumab are approved for the treatment of ulcerative colitis. ${ }^{1-6}$

Since the pro-inflammatory cytokine TNF- $\alpha$ plays an important role in host defence, treatment with TNF- $\alpha$ inhibitors has been subject to extensive post-marketing safety assessment, including the risk of infections. Studies assessing the risk of serious infections in people treated with TNF- $\alpha$ inhibitors for rheumatoid arthritis have gradually revealed a largely coherent pattern of a moderately increased risk of serious infections in the initial phase of treatment and a subsequent decline in risk. ${ }^{7-10}$ Data are, however, less clear when it comes to the risk of serious infections in people treated with TNF- $\alpha$ inhibitors for inflammatory bowel diseases. A meta-analysis based on 22 randomised controlled trials ${ }^{11}$ and a pooled analysis of 10 randomised controlled trials ${ }^{12}$ did not suggest an increased risk of serious infections in people with inflammatory bowel disease treated with TNF- $\alpha$ inhibitors compared with placebo. However, randomised controlled trials often represent selected patient populations, which is why post-marketing observational studies are essential to evaluate safety in a real world setting. A register based cohort study of people with inflammatory bowel disease did not find an increased risk of serious infections associated with TNF- $\alpha$ inhibitor treatment compared with propensity score matched patients treated with thiopurines. ${ }^{13}$ 
However, another register based study reported an increased risk of serious infections associated with infliximab use in people with inflammatory bowel disease, ${ }^{14}$ as did a study based on data from the Food and Drug Administration Adverse Event Reporting System. ${ }^{15}$ Thus the risk of infections associated with use of TNF- $\alpha$ inhibitors in people with inflammatory bowel disease is unclear. We conducted a nationwide population based cohort study using linked registry data to investigate the risk of serious infections in Danish people with inflammatory bowel disease treated with TNF- $\alpha$ inhibitors.

\section{Methods}

Using the Danish civil registration system, ${ }^{16}$ which contains information on the sex, date of birth, and vital status of all Danish citizens, we identified a source population, including all individuals aged 15-75 years living in Denmark between 2002 and 2012. By use of the unique personal identification number assigned to all Danish citizens at birth, we could link the source population to other national registries. From the national patient registry, ${ }^{17}$ a registry containing information on all hospital admissions in Denmark since 1977, and since 1995 extended to include all outpatient visits and emergency room contacts, we identified people with inflammatory bowel disease from ICD-8 and ICD-10 codes (international classification of diseases, eighth and 10th revisions, respectively): ICD-8 codes 56300-02 and 56308-09 and ICD-10 code K50 for Crohn's disease; ICD- 8 codes 56319 and 56309 and ICD-10 code K51 for ulcerative colitis. Furthermore, we used the national patient registry to ascertain history of comorbidities and study outcomes based on ICD-10 codes.

From the Danish drug prescription registry, ${ }^{18}$ established in 1995 and containing individual level information on all prescriptions redeemed at Danish pharmacies, we obtained data on drugs. Although the treatment with TNF- $\alpha$ inhibitors for inflammatory bowel disease were introduced in Denmark in 1999, we started the study in 2002 and excluded people who used TNF- $\alpha$ inhibitors before the start of the study; in this way, early drug users who were treated in the first years after the introduction of TNF- $\alpha$ inhibitors (who are likely to be different from the drugs' eventual stable user population, in terms of factors such as disease severity and therefore may introduce bias ${ }^{19}$ ) were not eligible for inclusion. We also excluded people with a diagnosis of HIV infection (ICD-10 code B20; ICD-8 codes 07983 and Y4149) and those with a history of organ or tissue transplantation (ICD-10 code Z94; ICD-8 not available).

\section{Drug use}

To assure completeness of data we obtained information on use of TNF- $\alpha$ inhibitors (including infliximab, adalimumab, and certolizumab pegol) from four sources: manually collected data on a nationwide cohort of all Danish people with inflammatory bowel disease treated with infliximab between its introduction in 1999 and 2005; data from the Danish Ministry of
Health, which after 2003 has kept track of TNF- $\alpha$ inhibitor treatment in all Danish hospital settings through procedure codes registered in the national patient registry; information on TNF- $\alpha$ inhibitor treatment reported to the Danish Crohn Colitis Database (a database designed to prospectively register Danish residents with inflammatory bowel disease ${ }^{20}$ ) during 2002-10; and information from the Danish drug prescription registry on prescriptions for adalimumab and certolizumab pegol during the study period (there are no data on infliximab use from this data source as intravenous infusion of the drug is confined to the hospital setting). Some patients were registered in more than one of the four sources; however, we considered one registration as sufficient to be defined as having used drugs. We defined patients as TNF- $\alpha$ inhibitor users from the date of first dose and onwards; thus we categorised patients as ever users once they had been "exposed" to the drug.

\section{Study outcomes}

The main outcome was any serious infections, defined as a diagnosis of infection associated with hospital admission (including primary and secondary discharge diagnoses) after cohort entry (outpatient diagnoses of infections were not included). Supplementary etable 1 shows the infection diagnoses included in the main outcome (any serious infection) and subdivided into six groups of site specific infections. These included respiratory tract infections, gastrointestinal infections, urological or gynaecological infections, skin and subcutaneous tissue infections, sepsis, and other infections (including opportunistic infections and tuberculosis).

\section{Propensity score model approach}

To take into account potential confounders we used propensity score methods. We calculated the propensity scores as the predicted probability of starting treatment with TNF- $\alpha$ inhibitors conditional on variables thought to be either confounders or predictors for the outcome. These variables included, among others, socioeconomic status; degree of urbanisation; comorbidities; concurrent drug use; history of gastrointestinal or anal fistula, abscess, or fissure; and gastrointestinal surgery (supplementary etable 2 presents a list of all variables included in the propensity score). In preliminary analyses we attempted propensity score adjustment on the full background cohort. However, because of substantial heterogeneity of risk estimates across propensity score strata (likely because the propensity score was highly discriminative (that is, a high $\mathrm{C}$ statistic) given substantial differences in characteristics between TNF- $\alpha$ inhibitor users and non-users), which violates the basic assumptions of this approach, it was abandoned. In the interest of minimising bias and obtaining reliable estimates from a valid model, we subsequently opted for a propensity score matching strategy. A well balanced match was considered as a standardised difference between users and non-users of TNF- $\alpha$ inhibitors below 10\% for any 
given variable. Firstly, we attempted matching in a ratio of 1:4 for users and non-users, respectively, but we found matches for less than half of the users and matching yielded only approximately two matches for each user and use groups that were poorly matched; we therefore abandoned this approach. Secondly, we attempted matching in a ratio of 1:1 for users and non-users, respectively, with all baseline characteristics included in the propensity score. This left a well balanced cohort; however, a substantial number of users could not be included owing to no appropriate matches. Therefore we removed the two variablesazathioprine use and oral corticosteroid use-that had the most discriminatory influence (large differences in prevalence between users and non-users and high standardised differences before matching) from the propensity score. Matching on this modified propensity score in a 1:1 ratio allowed a larger number of TNF- $\alpha$ inhibitors users (and corresponding non-user matches) to be included while maintaining a cohort well balanced on all matching characteristics; we therefore selected this as the final model (supplementary efigure illustrates the standardised differences before and after matching on propensity scores). The strategy for incorporation of propensity scores is described below and the two variables that were removed from the propensity score were instead adjusted for in a multivariable Cox regression model.

\section{Statistical analyses}

The matching process was done in two consecutive stages. Firstly, to remove users of TNF- $\alpha$ inhibitors with no potential matches, we matched on the following fixed factors representing key characteristics; sex, age (in five year intervals), inflammatory bowel disease type (Crohn's disease or ulcerative colitis), and time since diagnosis ( $<1,1-4,5-9,10-19$, and $\geq 20$ years) in a 1:n variable ratio. Next, by use of logistic regression, we estimated the individual propensities for initiating TNF- $\alpha$ inhibitor treatment. We chose the nearest neighbor caliper matching method, as this method seems to generate the most precise matching with lowest potential for bias and is appropriate in relatively small cohorts. ${ }^{21}$ Pairs of TNF- $\alpha$ inhibitor users and non-users with nearest propensity scores were matched (ratio 1:1) based on the logit of the propensity score with a caliper width of $0.2^{22}$ of the standard deviation of the propensity score. In addition we assigned non-users the date of the first day of TNF- $\alpha$ inhibitor use of their user matches as their first day of follow-up.

Using Cox proportional hazards models, we estimated hazard ratios with 95\% confidence intervals for serious infections in propensity score matched users versus non-users of TNF- $\alpha$ inhibitors, with days since the start of treatment as the underlying timescale. We assessed the risk of any serious infection in two risk periods, of 90 days and 365 days, and additionally in subgroups according to sex and inflammatory bowel disease subtype. Risk estimates of site specific infections were assessed in the 365 days risk period alone (owing to limited power for the 90 day risk period).
Furthermore, we performed a subanalysis estimating hazard ratios according to accumulated number of TNF- $\alpha$ inhibitor doses compared with non-users. Patients were followed from cohort entry until the date of first diagnosis of infection, loss to follow-up (emigration or disappearance), death, end of study (31 December 2012), or end of follow-up, whichever event occurred first. Risk estimates were adjusted for oral corticosteroid use and azathioprine use at baseline, as these variables were not included in the propensity score.

We did some supplementary analyses. To test for confounding by indication, we included an analysis with an active comparator drug. We compared the risk of infections in patients who started TNF- $\alpha$ inhibitors with those who started azathioprine. Although these two drugs are often used in combination in practice, in the interest of isolating associations attributable to one specific drug, in this analysis we only included patients who started monotherapy with the respective drug and had no history of use of the other drug-that is, patients starting TNF- $\alpha$ inhibitors had no history of azathioprine use and vice versa. Furthermore, we estimated the effects of a potential unmeasured or incompletely measured confounder on the main outcome, by using the array approach described by Schneeweiss. ${ }^{23}$

Because risk estimates might be more precise when assessing the risk of an adverse event while receiving treatment, we conducted an analysis with a time varying drug use definition (divided into current and past use and compared with non-users). Reliable data on specific TNF- $\alpha$ inhibitors were available only from one of the drug sources (the national patient registry) and only for 2005 and onwards; hence the analysis was based on a subcohort with data on TNF- $\alpha$ inhibitor use from the national patient registry during this period. Patients contributed follow-up as current users in a period set from the date of each administered dose and as long as a newly administered TNF- $\alpha$ inhibitor was registered in a TNF- $\alpha$ inhibitor drug specific interval (eight weeks for infliximab, two weeks for adalimumab, and four weeks for certolizumab pegol, in accordance with current treatment guidelines). We allowed for a gap of up to four weeks between the end of the interval of the latest dose and the date of the subsequent dose being administered to account for irregularities in the dosage interval. Lastly, because matching on the propensity score had led to the exclusion of a large number of patients who used TNF- $\alpha$ inhibitors, we did an analysis estimating hazard ratios of the main outcome based on the entire cohort matched on fixed factors, but not on propensity scores.

To confirm the proportional hazards model assumptions, we tested for martingale residuals for study exposure and the two baseline adjusted variables (azathioprine use and corticosteroid use). Based on visual inspection, no violation of the assumptions was detected. We imputed data for the one variable that had missing values $(0.8 \%$ missing for socioeconomic status; see supplementary etable 2 ) using the mode value. Loss to follow-up was negligible (1.1\%) and therefore not further assessed. 
Analyses were carried out using SAS version 9.4 (Cary, NC). We considered differences to be statistically significant when the $95 \%$ confidence intervals did not overlap 1.0 or when the $P$ value was $<0.05$ (all tests were two sided).

\section{Patient involvement}

There was no patient involvement in this study.

\section{Results}

From the source population of 4160526 people, we identified 53252 people with inflammatory bowel disease (fig 1). A total of $860(2 \%)$ patients were excluded owing to a diagnosis of HIV/AIDS, history of organ or tissue transplantation, or use of tumour necrosis factor- $\alpha$ (TNF- $\alpha$ ) inhibitors before entry to the cohort. This left a background cohort of 52392 people with inflammatory bowel disease, among whom 4300 received TNF- $\alpha$ inhibitors. In the background cohort the patients who used TNF- $\alpha$ inhibitors were characterised by having a younger median age, higher proportion with Crohn's disease, and a higher prevalence of intestinal surgery and inflammatory bowel disease related drug use compared with cohort members who were non-users (see supplementary etable 3 ).

Matching on fixed factors (84 TNF- $\alpha$ inhibitor users were excluded because of no potential match) and propensity scores (2673 TNF- $\alpha$ inhibitor users were excluded because of no potential match) yielded 1543 patients who used TNF- $\alpha$ inhibitors (infliximab, $\mathrm{n}=1243$; adalimumab, $\mathrm{n}=201$; certolizumab pegol, $\mathrm{n}=3$, unspecified, $\mathrm{n}=96$ ) and 1543 non-users who were included in the study cohort. Users of TNF- $\alpha$ inhibitors in the matched cohort had a mean age of 44.6 (SD 14.5) years, 57\% were women, and 57\% had ulcerative colitis

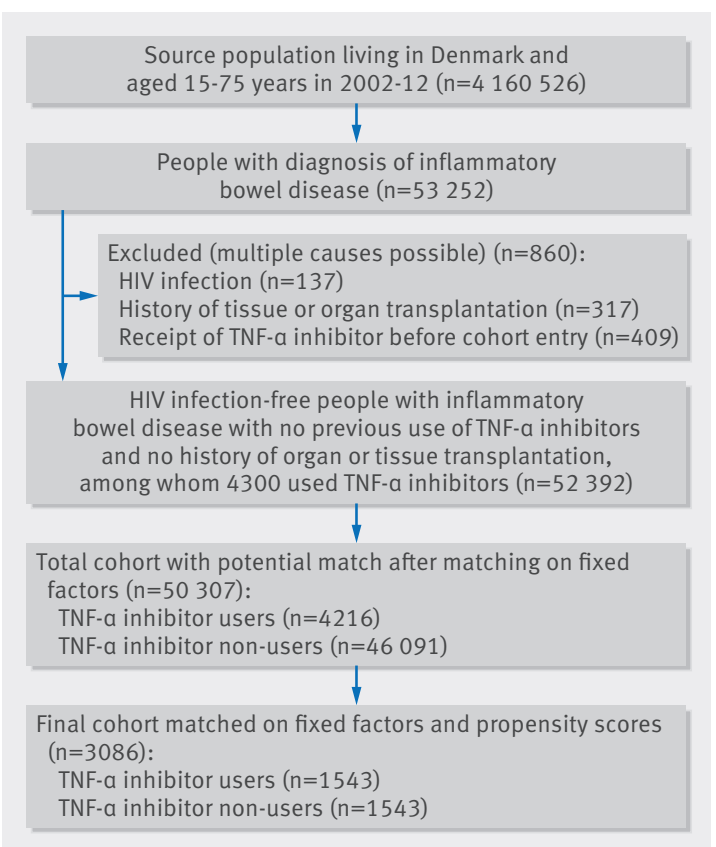

Fig 1 | Formation of study cohort. Fixed factors included age, sex, duration of disease, and inflammatory bowel disease subtype. TNF- $\alpha$ inhibitors=tumour necrosis factor inhibitors (table 1). The study groups were well balanced on all baseline characteristics included in the propensity score and as fixed matching variables; oral corticosteroid use and azathioprine use were more common among patients treated with TNF- $\alpha$ inhibitors (table 1 and supplementary efigure 1).

\section{Risk of any serious infection}

Table 2 shows hazard ratios of any serious infections, comparing users of TNF- $\alpha$ inhibitors with matched non-users. Within 90 days after the start of treatment 51 cases of serious infections were observed in TNF- $\alpha$ inhibitor users (incidence 14/100 person years, 95\% confidence interval 11 to 18) compared with 33 (incidence $9 / 100$ person years, 6 to 13) in non-users, yielding a hazard ratio of 1.63 (95\% confidence interval 1.01 to 2.63). Subgroup analyses revealed a significantly increased hazard ratio for women $(2.08$, 95\% confidence interval 1.09 to 3.96) but not for men (1.20, 0.57 to 2.50). Point estimates were nominally increased for both ulcerative colitis and Crohn's disease (1.83, 0.96 to 3.47 and $1.39,0.67$ to 2.88 , respectively).

Within the 365 days risk period, 107 cases of serious infections (incidence 8/100 person years, 95\% confidence interval 7 to 10) were observed in users of TNF- $\alpha$ inhibitors compared with 78 (incidence 6/100 person-years, 5 to 7 ) in non-users. The hazard ratio was 1.27 (95\% confidence interval 0.92 to 1.75). For men the hazard ratio was 1.18 (0.73 to 1.91) and for women it was 1.34 (0.87 to 2.08). The hazard ratio was nominally increased for ulcerative colitis $(1.45,0.92$ to 2.27$)$ but not for Crohn's disease (1.05, 0.65 to 1.69).

In the subanalysis of accumulated number of TNF- $\alpha$ inhibitor doses, the risk of serious infections was significantly increased in patients receiving a single dose (hazard ratio 1.64, 95\% confidence interval 1.06 to 2.53) and decreased thereafter in those receiving two or three doses (1.18, 0.79 to 1.78$)$ and four doses or more (1.06, 0.66 to 1.69 , fig 2 ).

\section{Risk of site specific serious infections}

Among the six site specific infection groups, the hazard ratio was above 2 for several of the subgroups, including sepsis (2.45, 95\% confidence interval 0.65 to 9.19), urological or gynaecological infections (2.31, 0.64 to 8.29), and skin and soft tissue infections (2.51, 1.23 to 5.12), but only reached statistical significance for the last category (table 3). Among the 107 cases of serious infections detected in users of TNF- $\alpha$ inhibitors, the most common infections were pneumonia (24\%) and gastroenteritis (17\%). For skin and soft tissue infections, the diagnoses were dominated by abscesses, carbuncles, and furuncles (35\%), erysipelas (19\%), and herpes infections (12\%) (see supplementary etable 4). No cases of tuberculosis were detected among patients who used TNF- $\alpha$ inhibitors.

\section{Supplementary analyses}

Several supplementary analyses were performed to check the robustness of results. In the head to head analysis comparing patients who used TNF- $\alpha$ inhibitor 
Table 1 | Characteristics of matched study cohort of tumour necrosis factor- $\alpha$ (TNF- $\alpha$ ) inhibitor users and non-users with inflammatory bowel disease. Values are numbers (percentages) of patients unless stated otherwise

\begin{tabular}{|c|c|c|}
\hline Characteristics & $\begin{array}{l}\text { Users of TNF- } \alpha \\
\text { inhibitors }(n=1543)\end{array}$ & $\begin{array}{l}\text { Non-users of TNF- } \alpha \\
\text { inhibitors }(n=1543)\end{array}$ \\
\hline Mean (SD) age (years) & $44.6(14.5)$ & $44.6(14.6)$ \\
\hline Male sex & $656(43)$ & $656(43)$ \\
\hline \multicolumn{3}{|c|}{ Inflammatory bowel disease subtype: } \\
\hline Crohn's disease & $667(43)$ & $667(43)$ \\
\hline Ulcerative colitis & $876(57)$ & $876(57)$ \\
\hline \multicolumn{3}{|l|}{ Disease duration (years): } \\
\hline$<1$ & $392(25)$ & $392(23)$ \\
\hline $1-4$ & $530(34)$ & $530(34)$ \\
\hline $5-9$ & $360(23)$ & $360(23)$ \\
\hline $10-19$ & $238(15)$ & $238(15)$ \\
\hline$\geq 20$ & $23(2)$ & $23(2)$ \\
\hline \multicolumn{3}{|l|}{ Place of birth: } \\
\hline Denmark & $1473(96)$ & $1477(96)$ \\
\hline Europe & $32(2)$ & $25(2)$ \\
\hline Other countries & $38(3)$ & $41(3)$ \\
\hline
\end{tabular}

Socioeconomic status

Employment:

\begin{tabular}{lll}
\hline Unknown, basic, or no qualifications & $560(36)$ & $618(40)$ \\
\hline Medium level qualifications & $212(14)$ & $200(13)$ \\
\hline High level qualifications & $146(10)$ & $151(10)$ \\
\hline Self employed or coworking spouse & $68(4)$ & $54(4)$ \\
\hline Outside labour market & $424(28)$ & $384(25)$ \\
\hline Pensioned & $133(9)$ & $136(9)$ \\
\hline
\end{tabular}

Degree of urbanisation

Population density (inhabitants per $\mathrm{km}^{2}$ ):

\begin{tabular}{lll}
\hline$\leq 49$ & $106(7)$ & $116(8)$ \\
\hline $50-99$ & $465(30)$ & $446(29)$ \\
\hline $100-199$ & $341(22)$ & $343(22)$ \\
\hline$\geq 200$ & $160(10)$ & $180(12)$ \\
\hline Copenhagen suburbs & $306(20)$ & $313(20)$ \\
\hline Copenhagen & $165(11)$ & $145(9)$ \\
\hline
\end{tabular}

Comorbidities

Gastrointestinal:

\begin{tabular}{lll}
\hline Surgery & $115(8)$ & $104(7)$ \\
\hline Intestinal, anal, rectal fissure, fistula, abscess & $34(2)$ & $25(2)$ \\
\hline Cardiovascular disease & $135(9)$ & $140(9)$ \\
\hline Chronic pulmonary disease & $86(6)$ & $77(5)$ \\
\hline Diabetes & $104(7)$ & $108(7)$ \\
\hline Liver disease & $23(2)$ & $18(1)$ \\
\hline Renal disease & $16(1)$ & $14(1)$ \\
\hline Rheumatic disease & $101(7)$ & $68(4)$ \\
\hline Cancer & $50(3)$ & $36(2)$ \\
\hline Previous infections* & $117(8)$ & $105(7)$ \\
\hline Non-inflammatory bowel disease drugst: & & \\
\hline Cardiovascular & $347(23)$ & $385(25)$ \\
\hline Antianemic & $231(15)$ & $221(14)$ \\
\hline Pulmonary antiobstructive & $173(11)$ & $172(11)$ \\
\hline Antimicrobials & $722(47)$ & $703(46)$ \\
\hline No of prescription drugs used in past 6 months: & & \\
\hline 0 & $110(7)$ & $86(6)$ \\
\hline 1 or 2 & $257(17)$ & $256(17)$ \\
\hline 3 or 4 & $244(16)$ & $278(18)$ \\
\hline$\geq 5$ & $932(60)$ & $923(60)$ \\
\hline Inflammatory bowel disease drugst & & \\
\hline 5 -aminosalicylic acid/sulfasalazine & $670(43)$ & $686(45)$ \\
\hline Intestinal corticosteroids & $283(18)$ & $300(19)$ \\
\hline Methotrexate, cyclosporine, cyclophosphamide & $42(3)$ & $20(1)$ \\
\hline Azathioprineł & $690(45)$ & $217(14)$ \\
\hline Oral corticosteroidsł & $861(56)$ & $303(20)$ \\
\hline & & \\
\hline & & \\
\hline & & \\
\hline
\end{tabular}

monotherapy with those using azathioprine monotherapy (see patient characteristics in supplementary etable 5), the hazard ratios for serious infections associated with use of TNF- $\alpha$ inhibitors were 2.17 (0.85 to 5.52) for the 90 day risk period and 2.05 (0.97 to 4.36) for the 365 day risk period (table 4). The effect of a potential unmeasured confounder was tested on the main outcome within the 90 day risk period, by assuming a wide range of combinations of confounder prevalence in the user group and strengths of the association between the confounder and infections (see supplementary etable 6). For example, assuming a confounder, such as smoking, was present in $30 \%$ of the TNF- $\alpha$ inhibitors user group and $20 \%$ of the non-user group, and if the confounder increased the risk of infections twofold, the observed point estimate of 1.63 would have been biased by $8 \%$ and the confounder adjusted estimate would be 1.50 . The analyses using an alternative time varying definition for drug use were based on a matched subcohort of 1472 people who used TNF- $\alpha$ inhibitors and 1472 non-user controls. Within 90 days of follow-up, the hazard ratio for current use was 1.70 (1.03 to 2.81) and was not estimable for past use because of no outcome events in this group. For the 365 day risk period, the hazard ratios were 1.29 (0.90 to 1.85) for current use and 1.20 (0.74 to 1.95) for past use (see supplementary etable 7). The analyses based on the cohort matched on fixed factors but not on propensity scores, including 4216 users of TNF- $\alpha$ inhibitors and 46091 non-users, yielded higher risk estimates but a similar risk pattern as the main analysis, with hazard ratios of 1.81 (1.45 to 2.24) for the 90 days risk period and 1.52 (1.33 to 1.74) for the 365 days risk period (see supplementary etable 8). Supplementary etable 9 shows the characteristics of users of TNF- $\alpha$ inhibitors excluded from the propensity score matched cohort. Excluded patients were characterised by having more severe inflammatory bowel disease, as witnessed by a higher prevalence of inflammatory bowel disease related drug use and surgery, but had a lower prevalence of general comorbidities and use of other drugs and were mostly patients with Crohn's disease.

\section{Discussion}

In this nationwide propensity score matched cohort study we found a statistically significant $63 \%$ increased risk of serious infections associated with TNF- $\alpha$ inhibitor treatment in people with inflammatory bowel disease within 90 days of the start of treatment. Assessing the risk of serious infection during a longer follow-up period of 365 days, the association was attenuated and was no longer significant. For site specific serious infections, we found increased point estimates for sepsis, urological or gynaecological infections, and skin and soft tissue infections; these analyses should, however, be interpreted cautiously because of limited power.

\section{Strengths and limitations of this study}

The primary strength of this study is the nationwide register based design, providing a cohort covering the entire Danish population with inflammatory bowel 


\begin{tabular}{|c|c|c|}
\hline Characteristics & $\begin{array}{l}\text { Users of TNF- } \alpha \\
\text { inhibitors ( }=1543)\end{array}$ & $\begin{array}{l}\text { Non-users of TNF- } \alpha \\
\text { inhibitors }(n=1543)\end{array}$ \\
\hline \multicolumn{3}{|c|}{ Healthcare use in past year } \\
\hline \multicolumn{3}{|c|}{ No of hospital admissions: } \\
\hline 0 & $872(57)$ & $915(60)$ \\
\hline 1 or 2 & $539(35)$ & $502(33)$ \\
\hline$\geq 3$ & $132(9)$ & $126(8)$ \\
\hline \multicolumn{3}{|c|}{ No of outpatient contacts: } \\
\hline 0 & $371(24)$ & $352(23)$ \\
\hline 1 or 2 & $944(61)$ & $1007(65)$ \\
\hline$\geq 3$ & $228(15)$ & $184(12)$ \\
\hline
\end{tabular}

The cohort was matched on fixed factors (sex, age, disease duration, and inflammatory bowel disease subtype) and propensity scores. All characteristics are as current at cohort entry unless stated otherwise.

*Defined as previous infections associated with hospital admission within one year before cohort entry. tAs registered within one year before cohort entry.

₹Not included in propensity score.

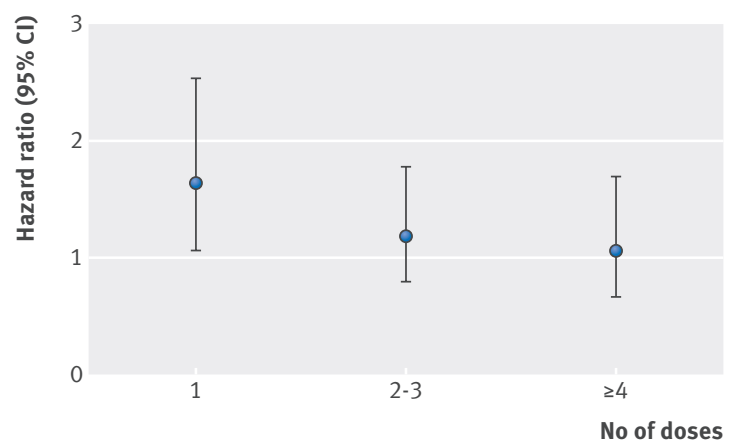

TNF- $a$ inhibitor users

No of patients 1543

No of events 35

1326

43

913

29

Each dose category is compared to TNF-a inhibitor non-users

Fig 2 | Risk of any serious infection according to accumulated number of tumour necrosis factor- $\alpha$ (TNF- $\alpha$ ) inhibitor doses in users compared with non-users. Error bars indicate $95 \%$ confidence intervals

disease with minimal loss to follow-up and independent ascertainment of exposure and outcomes. A validation study with assessment of nearly 800 patients estimated the completeness of registration of inflammatory bowel disease in the national patient registry to be $94 \%$, whereas the estimated validity, expressed as the proportion of confirmed diagnoses in the registry, was $97 \%$ for Crohn's disease and $90 \%$ for ulcerative colitis. ${ }^{24}$ A complete treatment history could be obtained for all patients treated with TNF- $\alpha$ inhibitors, which allowed follow-up from the start of treatment hence avoiding prevalent user bias. ${ }^{25}$ Furthermore, we believe that our outcome definition (infections associated with hospital admission) is properly suited to identify serious infections that are potentially life threatening (as opposed to an outcome definition that also includes outpatient diagnoses).

The study also has limitations. In the interest of internal validity of the analyses, that is to minimise bias, we applied matching on fixed variables and propensity scores. Use of TNF- $\alpha$ inhibitors was relatively uncommon in Denmark during the study period ( $9 \%$ of the background cohort were treated with the drugs); this implies that patients with the most severe disease were those who received this treatment. Finding adequate non-user matches for these patients was difficult, as ideal controls should have the same distribution of characteristics to enable the estimation of unbiased risk. This phenomenon was mirrored by the fact that a relatively low number of patients who were treated with TNF- $\alpha$ inhibitors in the background cohort could be included in the final matched cohort. Thus, while our matching method maximised internal validity, it may potentially have influenced the generalisability of the results. Therefore we performed a supplementary analysis including the majority of users and non-users of TNF- $\alpha$ inhibitors (only a few patients were excluded as patients were only matched on fixed factors, such as

\begin{tabular}{|c|c|c|c|c|c|}
\hline \multirow[b]{2}{*}{ Variables } & \multicolumn{2}{|c|}{ Users of TNF- $\alpha$ inhibitors } & \multicolumn{2}{|c|}{ Non-users of TNF- $\alpha$ inhibitors } & \multirow{2}{*}{$\begin{array}{l}\text { Hazard ratio } \\
(95 \% \mathrm{Cl})\end{array}$} \\
\hline & No of patients & No of events & No of patients & No of events & \\
\hline \multicolumn{6}{|l|}{90 days risk period } \\
\hline Main analysis & 1543 & 51 & 1543 & 33 & 1.63 (1.01 to 2.63$)$ \\
\hline \multicolumn{6}{|l|}{ Sex: } \\
\hline Men & 656 & 17 & 656 & 17 & $1.20(0.57$ to 2.50$)$ \\
\hline Women & 887 & 34 & 887 & 16 & 2.08 (1.09 to 3.96$)$ \\
\hline \multicolumn{6}{|c|}{ Inflammatory bowel disease subtype: } \\
\hline Ulcerative colitis & 876 & 32 & 876 & 18 & 1.83 (0.96 to 3.47 ) \\
\hline Crohn's disease & 667 & 19 & 667 & 15 & 1.39 (0.67 to 2.88$)$ \\
\hline \multicolumn{6}{|l|}{365 days risk period } \\
\hline Main analysis & 1543 & 107 & 1543 & 78 & $1.27(0.92$ to 1.75$)$ \\
\hline \multicolumn{6}{|l|}{ Sex: } \\
\hline Men & 656 & 48 & 656 & 36 & $1.18(0.73$ to 1.91$)$ \\
\hline Women & 887 & 59 & 887 & 42 & 1.34 (0.87 to 2.08) \\
\hline \multicolumn{6}{|c|}{ Inflammatory bowel disease subtype: } \\
\hline Ulcerative colitis & 876 & 67 & 876 & 38 & 1.45 (0.92 to 2.27$)$ \\
\hline Crohn's disease & 667 & 40 & 667 & 40 & 1.05 (0.65 to 1.69$)$ \\
\hline
\end{tabular}




\begin{tabular}{|c|c|c|c|c|c|}
\hline Infection sites & \multicolumn{2}{|c|}{ Users of TNF- $\alpha$ inhibitors } & \multicolumn{2}{|c|}{ Non-users of TNF- $\alpha$ inhibitors } & $\begin{array}{l}\text { Hazard ratio } \\
(95 \% \mathrm{CI})\end{array}$ \\
\hline Gastrointestinal & 1543 & 28 & 1543 & 24 & $1.01(0.55$ to 1.86$)$ \\
\hline Respiratory tract & 1543 & 30 & 1543 & 28 & $0.75(0.42$ to 1.34$)$ \\
\hline Sepsis & 1543 & 6 & 1543 & 4 & 2.45 (0.65 to 9.19) \\
\hline Other & 1543 & 8 & 1543 & 5 & $1.24(0.36$ to 4.30$)$ \\
\hline
\end{tabular}

age and sex, and not on propensity scores) and found estimates to reveal a similar pattern as in our main analysis, although the magnitude of association was stronger in the supplementary analysis. This would be consistent with the notions that the population included in the main analysis is representative of the overall background population and that propensity score matching offered substantial control for confounders.

To further assess the impact of disease severity as a confounder, we compared the risk of infection between patients who used TNF- $\alpha$ inhibitors and those who used azathioprine; although this analysis was based on a low number of patients, results did not indicate confounding by indication. We used the array approach to test for residual confounding due to unmeasured or incompletely measured confounders, which showed that such a confounder would have to be strongly associated with the study outcome and that the difference in prevalence of such a confounder between users and non-users would have to be rather large to explain the observed point estimate. For instance, adjustment for a confounder that increases the risk of serious infections twofold and with an absolute prevalence difference of $10 \%$ between users and non-users of TNF- $\alpha$ inhibitors would only marginally attenuate the observed point estimate. However, the observed lower bound of the $95 \%$ confidence limit was close to 1.0; thus, adjustment for the confounder in the hypothetical scenario would likely render results non-significant.

Although we defined drug use according to the intention to treat principle (ever or never users) in our main analysis, assessment of the risk of serious infections while receiving treatment might give more precise risk estimates. We therefore did a robustness analysis using a time varying definition for drug exposure. Overall, the risk estimates for current use in this analysis were similar to those in the main analysis. Owing to limitations in power, it was not possible to conclude whether there were differences between the current and past use categories.

Additionally, the ability of our study to draw conclusions on specific infections was limited because of issues with power. Several point estimates were high but not statistically significantly increased. However, the absence of significant associations does not provide evidence that risk is not increased. Lastly, our study was not powered to study either opportunistic infections as a separate outcome, cause specific death, or the different TNF- $\alpha$ inhibitors separately.

\section{Comparison to other studies}

In previous population based studies, the answer as to whether TNF- $\alpha$ inhibitor treatment is associated with an increased risk of serious infections in people with inflammatory bowel disease has been conflicting. ${ }^{13} 142627$ A study from the American TREAT (Crohn's Therapy, Resource, Evaluation, and Assessment Tool) registry assessed the risk of serious infections among 2853 patients treated with infliximab for Crohn's disease, with a mean follow-up time of 5.2 years $^{14}$ and found that treatment was an independent risk factor for serious infections (hazard ratio 1.43, 95\% confidence interval 1.11 to 1.84). Another register based cohort study based on the SABER (Safety Assessment of Biologic Therapy) initiative ${ }^{13}$ examined rates of serious infection in 2323 people treated with TNF- $\alpha$ inhibitors for inflammatory bowel disease compared with propensity score matched patients receiving non-biological comparator drugs (azathioprine/mercaptopurine). This study found no significantly increased risk of infections associated with TNF- $\alpha$ inhibitor treatment (hazard ratio 1.10, 95\% confidence interval 0.83 to 1.46 ); with a similar risk throughout the year of follow-up and hence no suggestion of an initially increased risk. In general, the variations in risk estimates observed in observational studies may be due to differences in the background rates of infections in the studied populations. For example, a high incidence rate of serious infections was observed

\begin{tabular}{|c|c|c|c|c|c|}
\hline \multirow[b]{2}{*}{ Risk period } & \multicolumn{2}{|c|}{ Users of TNF- $\alpha$ inhibitors } & \multicolumn{2}{|c|}{ Users of azathioprine } & \multirow{2}{*}{$\begin{array}{l}\text { Hazard ratio } \\
(95 \% \mathrm{Cl})\end{array}$} \\
\hline & No of patients & No of events & No of patients & No of events & \\
\hline 90 days & 268 & 12 & 268 & 7 & 2.17 (0.85 to 5.52$)$ \\
\hline 365 days & 268 & 18 & 268 & 11 & 2.05 (0.97 to 4.36$)$ \\
\hline
\end{tabular}


in the comparison group in the SABER cohort, probably as a result of the predominance of low income and vulnerable patients in the cohort and the findings may therefore primarily be applicable to this population. In our study we were able to obtain data on the entire Danish population with inflammatory bowel disease where everyone has equal access to medical care and treatment (including with TNF- $\alpha$ inhibitors); hence we believe our observations apply to the broader population with inflammatory bowel disease.

We observed an increased risk after 90 days of follow-up that declined within 365 days following the start of treatment with TNF- $\alpha$ inhibitors as well as decreasing hazard ratios with increasing number of accumulated TNF- $\alpha$ inhibitor doses. This risk pattern resembles that suggested by several studies evaluating the association between TNF- $\alpha$ inhibitors and risk of serious infections in people with rheumatoid arthritis. ${ }^{7-10}$ The decline in risk over time may be because TNF- $\alpha$ inhibitor treatment actually causes early, but not late, infections in susceptible people. However, it may also partly be explained by detection bias, as clinicians may have a lower threshold for identifying infections early in the course of TNF- $\alpha$ inhibitor treatment. Lastly, it may be due to a depletion of people susceptible to infection (that is, high risk people have infections early and discontinue treatment, hence leaving a cohort that is at lower risk of infection); these theories need further investigation.

The significantly increased risk of skin and soft tissue infections observed in our study is not unexpected, as an association between TNF- $\alpha$ inhibitor treatment and skin and soft tissue infections has been shown previously. ${ }^{2829}$ However, although power was limited, our analyses of site specific infections indicated a possible increased risk of not only skin and soft tissue infections but also several other infection types. Considering the central role of TNF- $\alpha$ inhibitors in host defence, susceptibility to infections in people treated with drugs inhibiting this cytokine is biologically plausible. However, whether the physiological role of TNF- $\alpha$ inhibitors in host defence varies between tissues leading to increased risk of some specific infections but not others is not clear and calls for further research.

\section{Conclusion}

In this population based nationwide cohort study of people with inflammatory bowel disease we found that use of TNF- $\alpha$ inhibitors was associated with a modestly increased risk of serious infections within the first 90 days after the start of treatment, which declined with prolonged follow-up. The results suggest that clinical vigilance of potential infectious complications is indicated in people treated with TNF- $\alpha$ inhibitors for inflammatory bowel disease, especially early in the course of treatment.

We thank Henrik Svanström, Department of Epidemiology Research, Statens Serum Institut, Copenhagen, Denmark, for additional statistical support, which was provided without compensation.

Contributors: NNA, BP, NFM, MA, and TJ conceived and designed the study. MA and TJ acquired the data. NNA, BP, MA, NFM, and TJ analysed and interpreted the data. NNA drafted the manuscript. BP,
MA, NFM, and TJ critically revised the manuscript for important intellectual content. MA did the statistical analysis. TJ and NNA obtained funding. BP and TJ supervised the study. NNA had full access to all of the data in the study and takes responsibility for the integrity of the data and the accuracy of the data analysis. She is the guarantor. Funding: This study was supported by grants from the Lundbeck Foundation (grant No R83-A7812), For Women in Science (by UNESCO and L'Oreal), the Beckett Foundation, and the Danish Colitis Crohn Association. TJ holds a female research leader grant (No 09-066323) from the Danish Council for Independent Research. BP is supported by the Danish Council for Independent Research (No 4092-00155). The funding agencies had no role in the design and conduct of the study; the collection, management, analysis, and interpretation of the data; the preparation, review, or approval of the manuscript; or the decision to submit the manuscript for publication.

Competing interests: All authors have completed the ICMJE uniform disclosure form at www.icmje.org/coi_disclosure.pdf and declare: TJ has received funding for travel and a speakers fee from AbbVie and NNA has received funding for travel and speakers fees from MSD and from Ferring; no financial relationships with any organisations that might have an interest in the submitted work in the previous three years; no other relationships or activities that could appear to have influenced the submitted work.

Ethical approval: The study was approved by the Danish Data Protection Agency (approval No 2008-54-0472). Ethical approval is not required for registry based research in Denmark.

Data sharing: No additional data available.

Transparency: The lead author (NNA) affirms that the manuscript is an honest, accurate, and transparent account of the study being reported; that no important aspects of the study have been omitted; and that any discrepancies from the study as planned (and, if relevant, registered) have been explained.

This is an Open Access article distributed in accordance with the Creative Commons Attribution Non Commercial (CC BY-NC 4.0) license, which permits others to distribute, remix, adapt, build upon this work non-commercially, and license their derivative works on different terms, provided the original work is properly cited and the use is noncommercial. See: http://creativecommons.org/licenses/by-nc/4.0/.

1 Colombel JF, Sandborn WJ, Rutgeerts P, et al. Adalimumab for maintenance of clinical response and remission in patients with Crohn's disease: the CHARM trial. Gastroenterology 2007;132:52-65.

2 Colombel JF, Sandborn WJ, Reinisch W, et al. Infliximab, azathioprine, or combination therapy for Crohn's disease. N Engl J Med 2010;362:1383-95

3 Hanauer SB, Feagan BG, Lichtenstein GR, et al. Maintenance infliximab for Crohn's disease: the ACCENT I randomised trial. Lancet 2002;359:1541-9.

4 Rutgeerts P, Sandborn WJ, Feagan BG, et al. Infliximab for induction and maintenance therapy for ulcerative colitis. N Engl I Med 2005;353:2462-76

5 Sandborn WJ, Feagan BG, Stoinov S, et al. Certolizumab pegol for the treatment of Crohn's disease. N Engl J Med 2007;357:228-38.

6 Sandborn WJ, Van AG, Reinisch W, et al. Adalimumab induces and maintains clinical remission in patients with moderate-to-severe ulcerative colitis. Gastroenterology 2012;142:257-65.

7 Askling J, Fored CM, Brandt L, et al. Time-dependent increase in risk of hospitalisation with infection among Swedish RA patients treated with TNF antagonists. Ann Rheum Dis 2007;66:1339-44.

8 Askling J, Dixon W. The safety of anti-tumour necrosis factor therapy in rheumatoid arthritis. Curr Opin Rheumatol 2008;20:138-44.

9 Bongartz T, Sutton AJ, Sweeting MJ, et al. Anti-TNF antibody therapy in rheumatoid arthritis and the risk of serious infections and malignancies: systematic review and meta-analysis of rare harmful effects in randomized controlled trials. JAMA 2006;295:2275-85

10 Listing J, Strangfeld A, Kary S, et al. Infections in patients with rheumatoid arthritis treated with biologic agents. Arthritis Rheum 2005;52:3403-12.

11 Peyrin-Biroulet L, Deltenre P, de Suray N, et al. Efficacy and safety of tumor necrosis factor antagonists in Crohn's disease: meta-analysis of placebo-controlled trials. Clin Gastroenterol Hepatol 2008;6:644-53.

12 Lichtenstein GR, Rutgeerts P, Sandborn WJ, et al. A pooled analysis of infections, malignancy, and mortality in infliximab-and immunomodulator-treated adult patients with inflammatory bowel disease. Am J Gastroenterol 2012;107:1051-63.

13 Grijalva CG, Chen L, Delzell E, et al. Initiation of tumor necrosis factor-alpha antagonists and the risk of hospitalization for infection in patients with autoimmune diseases. JAMA 2011:306:2331-9.

14 Lichtenstein GR, Feagan BG, Cohen RD, et al. Serious infection and mortality in patients with Crohn's disease: more than 5 years of follow-up in the TREAT registry. Am J Gastroenterol 2012;107:1409-22. 
15 Deepak P, Stobaugh DJ, Ehrenpreis ED. Infectious complications of TNF-alpha inhibitor monotherapy versus combination therapy with immunomodulators in inflammatory bowel disease: analysis of the Food and Drug Administration Adverse Event Reporting System. J Gastrointestin Liver Dis 2013;22:269-76.

16 Pedersen CB, Gotzsche H, Moller JO, et al. The Danish Civil Registration System. A cohort of eight million persons. Dan Med Bull 2006;53:441-9.

17 Andersen TF, Madsen M, Jorgensen J, et al. The Danish National Hospital Register. A valuable source of data for modern health sciences. Dan Med Bull 1999;46:263-8

18 Kildemoes HW, Sorensen HT, Hallas J. The Danish National Prescription Registry. Scand I Public Health 2011:39(7 Suppl):38-41.

19 Schneeweiss S, Gagne JJ, Glynn RJ, et al. Assessing the comparative effectiveness of newly marketed medications: methodological challenges and implications for drug development. Clin Pharmacol Ther 2011:90:777-90.

20 The Danish Crohn Colitis Database (DCCD). 2011. www.dccd-ibd.dk। en \index.php.

21 Rassen IA, Shelat AA, Myers J, et al. One-to-many propensity score matching in cohort studies. Pharmacoepidemiol Drug Saf 2012;21(Suppl 2):69-80

22 Austin PC. Some methods of propensity-score matching had superior performance to others: results of an empirical investigation and Monte Carlo simulations. Biom / 2009;51:171-84.

23 Schneeweiss S. Sensitivity analysis and external adjustment for unmeasured confounders in epidemiologic database studies of therapeutics. Pharmacoepidemiol Drug Saf 2006;15:291-303.
24 Fonager K, Sorensen HT, Rasmussen SN, et al. Assessment of the diagnoses of Crohn's disease and ulcerative colitis in a Danish hospital information system. Scand / Gastroenterol 1996:31:154-9.

25 Schneeweiss S. A basic study design for expedited safety signal evaluation based on electronic healthcare data. Pharmacoepidemiol Drug Saf 2010;19:858-68

26 Ljung T, Karlen P, Schmidt D, et al. Infliximab in inflammatory bowel disease: clinical outcome in a population based cohort from Stockholm County. Gut 2004:53:849-53.

27 Schneeweiss S, Korzenik J, Solomon DH, et al. Infliximab and other immunomodulating drugs in patients with inflammatory bowel disease and the risk of serious bacterial infections. Aliment Pharmacol Ther 2009;30:253-64.

28 Dixon WG, Watson K, Lunt M, et al. Rates of serious infection, including site-specific and bacterial intracellular infection, in rheumatoid arthritis patients receiving anti-tumor necrosis factor therapy: results from the British Society for Rheumatology Biologics Register. Arthritis Rheum 2006;54:2368-76.

29 Galloway JB, Mercer LK, Moseley A, et al. Risk of skin and soft tissue infections (including shingles) in patients exposed to anti-tumour necrosis factor therapy: results from the British Society for Rheumatology Biologics Register. Ann Rheum Dis 2013;72:229-34.

(C) BMJ Publishing Group Ltd 2015

Supplementary information: efigure and etables 1-9 